\title{
Middle East Respiratory Syndrome coronavirus vaccine development: updating clinical studies using platform technologies
}

\author{
Jung-ah Choi and Jae-Ouk Kim ${ }^{*}$ \\ Science Unit, International Vaccine Institute, Seoul 08826, Republic of \\ Korea \\ (Received Oct 20, 2021 / Received Dec 12, 2021 / Accepted Dec 15, 2021)
}

Middle East Respiratory Syndrome coronavirus (MERS-CoV), a contagious zoonotic virus, causes severe respiratory infection with a case fatality rate of approximately $35 \%$ in humans. Intermittent sporadic cases in communities and healthcare facility outbreaks have continued to occur since its first identification in 2012. The World Health Organization has declared MERS-CoV a priority pathogen for worldwide research and vaccine development due to its epidemic potential and the insufficient countermeasures available. The Coalition for Epidemic Preparedness Innovations is supporting vaccine development against emerging diseases, including MERS-CoV, based on platform technologies using DNA, mRNA, viral vector, and protein subunit vaccines. In this paper, we review the usefulness and structure of a spike glycoprotein as a MERS$\mathrm{CoV}$ vaccine candidate molecule, and provide an update on the status of MERS-CoV vaccine development. Vaccine candidates based on both DNA and viral vectors coding MERSCoV spike gene have completed early phase clinical trials. A harmonized approach is required to assess the immunogenicity of various candidate vaccine platforms. Platform technologies accelerated COVID-19 vaccine development and can also be applied to developing vaccines against other emerging viral diseases.

Keywords: Middle East Respiratory Syndrome coronavirus (MERS-CoV), vaccine, platform technologies

\section{Introduction}

Middle East Respiratory Syndrome coronavirus (MERS-CoV) causes infectious respiratory diseases in humans (van Boheemen et al., 2012). The clinical features of MERS-CoV infection can vary from asymptomatic or mild illness to death due to pneumonia and multiorgan failure, especially in people with underlying comorbidities (Zumla et al., 2015). At the

${ }^{*}$ For correspondence. E-mail: jokim@ivi.int; Tel.: +82-2-881-1318; Fax: +82-2-881-1298

Copyright (c) 2022, The Microbiological Society of Korea end of October 2021, a total of 2,578 laboratory-confirmed cases of MERS, including 888 associated deaths, were reported globally since its first identification in the Kingdom of Saudi Arabia in 2012, with a case-fatality ratio (CFR) of $34.4 \%$. Most of these cases were reported in Saudi Arabia: 2178 cases, including 810 related deaths (CFR 37.2\%). The largest outbreaks occurred in Riyadh and Jeddah in Saudi Arabia in 2014 (Memish et al., 2020). The MERS outbreak outside the Middle East was reported in the Republic of Korea in 2015, with 186 laboratory-confirmed cases (185 cases in the Republic of Korea and 1 case in China) and 38 deaths (CFR 20.4\%) that were spread from only one male returning from the Arabian Peninsula (Cho et al., 2016). To date, intermittent sporadic cases among community clusters and healthcare facility outbreaks of MERS-CoV continue to occur (Memish et al., 2020). Since 2015, MERS-CoV has been indicated as an infectious pathogen prioritized by the WHO R\&D Blueprint for research and development in public health due to its epidemic potential and insufficient countermeasures (Mehand et al., 2018). The Coalition for Epidemic Preparedness Innovations (CEPI) (Røttingen et al., 2017), a global partnership formally founded in 2017 to accelerate the development of vaccines against emerging infectious disease and, therefore, establish investigational vaccine stockpiles before epidemics emerge, decided to fund the platform technology research for vaccine development focusing on MERS-CoV, Lassa fever, and Nipah virus in 2018 among diseases in the WHO R\&D Blueprint list (Plotkin, 2017; Maslow, 2018; Wong and Qiu, 2018; Gouglas et al., 2019) (Table 1). Later, the Chikungunya virus, Rift Valley Fever, and SARS-CoV-2 were also included for the CEPI investment (Bernasconi et al., 2020).

Platform technology refers to a basic manufacturing system, such as DNA, mRNA, viral vectors, and proteins, that can be applied to various vaccine antigens. Once platform techniques are established, the same basic components can be rapidly adopted for various vaccines by inserting new genetic codes or proteins from other pathogens. As data on a new platform accumulate over time, regulatory authorities will likely become familiar with moving new vaccines to the same platform in clinical trials and can expedite the approval of novel vaccines against emerging epidemics or pandemics. In addition, platform technologies will facilitate quicker reactions to emerging variants of the same pathogen. CEPI has accelerated the development and manufacture of vaccines against unknown pathogens within 16 weeks of antigen identification (Vandeputte et al., 2021). These platform technologies used in the development of MERS-CoV vaccines have led to a 
Table 1. Platform technologies for vaccines against emerging viral diseases funded by the Coalition for Epidemic Preparedness Innovations in 2018

\begin{tabular}{lll}
\hline \multicolumn{1}{c}{ Disease } & \multicolumn{1}{c}{ Partners } \\
\hline & Adenovirus vector & Janssen \& University of Oxford \\
MVA & Measles & IDT Biologika \\
DNA & Themis Bioscience \\
& Protein Subunit & Inovio Pharmaceuticals \\
& Adenovirus vector & The University of Queensland \\
\hline VSV & Janssen \& University of Oxford \\
& VSV & Profectus Biosciences, Emergent Biosolutions and PATH \\
Measles & International AIDS Vaccine Initiative (IAVI) \\
& DNA & Themis Bioscience \\
& RNA & Inovio Pharmaceuticals \\
& Adenovirus vector & CureVac \\
\hline \multirow{3}{*}{ Nipah } & Measles & Janssen \& University of Oxford \\
& Protein Subunit & The University of Tokyo \\
\hline
\end{tabular}

This Table was presented at a Coalition for Epidemic Preparedness Innovations workshop in Oslo, Norway in 2019.

COVID-19 vaccine against severe acute respiratory syndrome coronavirus 2 (SARS-CoV-2) (Kashte et al., 2021). In this paper, a spike that is a MERS-CoV vaccine candidate molecule for neutralizing targets and the status of MERS-CoV vaccine development using several platforms will be reviewed.

\section{MERS-CoV Spike as a Vaccine Target}

Genome sequence analysis has shown that MERS-CoV belongs to the Betacoronavirus genus of the Coronaviridae family, which includes bat SARS-like coronavirus, human coronavirus (HCoV-OC43, HCoV-HKU1, SARS-CoV, and

(A)

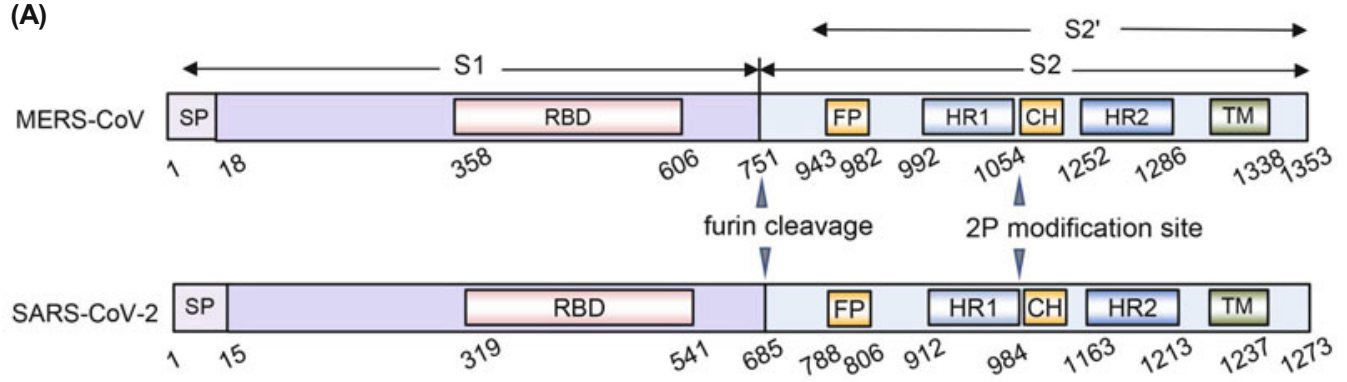

(B)

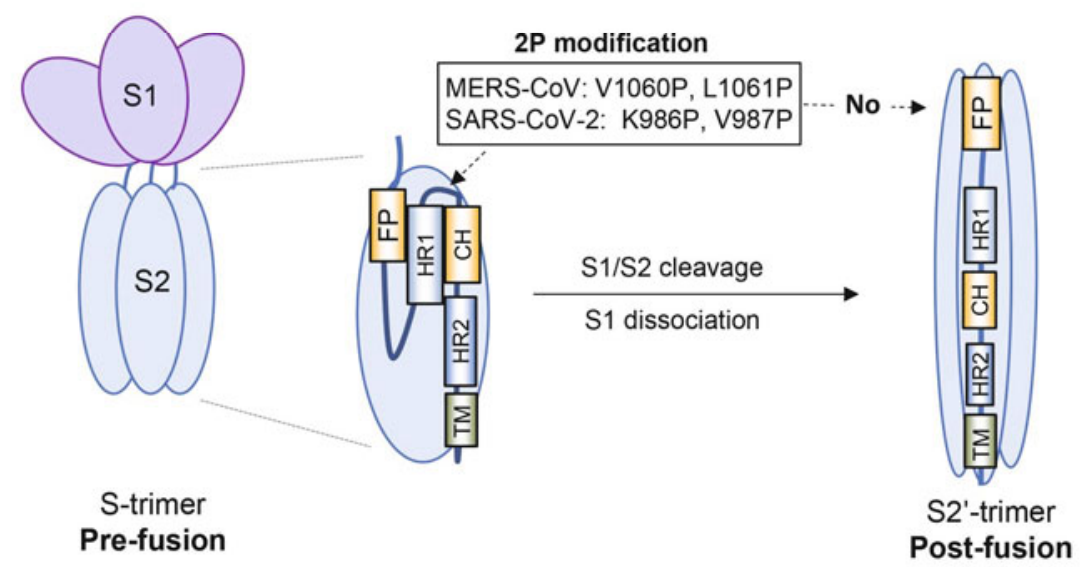

Fig. 1. MERS-CoV Spike as a vaccine target. (A) Region between HR1 and $\mathrm{CH}$ unfolds after $\mathrm{S}$ binds to human dipeptidyl peptidase 4, the host cellular receptor, and human angiotensin-converting enzyme 2 (hACE2) via the RBD of MERS-CoV and SARS-CoV-2, respectively. (B) Cleavage at S1/S2 and S2' sites causes irreversible structural changes from the pre-fusion to the post-fusion form. Replacement of two residues between HR1 and CH (V1060 and L1061 for MERS-CoV S; K986 and V987 for SARS-CoV-2) with proline stabilized S protein in the pre-fusion form. Prefusion S is beneficial for inducing neutralizing antibodies. RBD, receptor-binding domain; FP, fusion peptide; $\mathrm{CH}$, central helix; HR, heptad repeat; TM, transmembrane domain. 
SARS-CoV-2) (Hulswit et al., 2019; Chen et al., 2020; Bhat et al., 2021). MERS-CoV is a zoonotic virus, and it is believed to have originated from bats and transmitted to camels (Hemida et al., 2017). MERS-CoV has been identified in dromedaries in the Middle East, Africa, and South Asia (Mohd et al., 2016). MERS-CoV can be transferred to humans from infected dromedary camels, and human-to-human transmission has been reported more frequently in healthcare facilities than among families living in the same household (Drosten et al., 2014).

Like other viruses belonging to Coronaviridae, MERS-CoV is an enveloped virus with a positive-sense single-stranded RNA genome (de Groot et al., 2013). It has four structural proteins: spike (S), envelope, membrane, and nucleocapsid, of which $S$ glycoprotein is mainly used as a vaccine candidate for inducing neutralizing antibodies (Al-Amri et al., 2017). The MERS-CoV S glycoprotein acts as a viral fusion protein that mediates attachment to the host receptor, which is human dipeptidyl peptidase 4 (hDPP4), and fusion of the viral and cellular membranes (Du et al., 2013). S is synthesized as a single polypeptide chain of 1395 amino acids and is cleaved into the receptor-binding subunit $\mathrm{S} 1$ and the membrane-fusion subunit $\mathrm{S} 2$ by host furin protease during the infection process (Wang et al., 2013, 2014; Yu et al., 2015). The $S 1$ and S2 subunits trimerize to form a prefusion spike $(\sim 600 \mathrm{kDa})$ with $\sim 25 \mathrm{~N}$-linked glycans per monomer (Pallesen et al., 2017). S1 and S2 remain noncovalently bound in the prefusion conformation (Bosch et al., 2003).

The $\mathrm{S} 1$ subunit comprises the apex of the $\mathrm{S}$ trimer, including the receptor-binding domains (RBDs), and stabilizes the prefusion state of the S2 fusion machinery, which is anchored in the viral membrane. The S2 subunit contains the fusion peptide, two heptad repeats, and a transmembrane domain, all of which are required to mediate fusion of the viral and host cell membranes. S is further cleaved by host proteases at the so-called "S2'" site located immediately upstream of the fusion peptide. This cleavage has been proposed to activate proteins for membrane fusion via large-scale, irreversible conformational rearrangements (Fig. 1) (Walls et al., 2017).

The prefusion-stabilized MERS-CoV S protein (MERS S-2P) with V1060P and L1061P mutations still demonstrated highaffinity binding to hDPP4 and a panel of MERS-CoV neutralizing antibodies. Moreover, MERS S-2P induced high titers of neutralizing antibodies in mice (Pallesen et al., 2017). Therefore, the successful production of pre-fusion-stabilized MERS-CoV S proteins will contribute to the development of efficient protective interventions against current and emerging variants (Fig. 1B).

A technique involving the use of a molecular clamp consisting of a stable trimerization motif of 80 aa in length derived from HIV-1 gp41 was applied to viral fusion proteins such as respiratory syncytial virus fusion protein (McLellan et al., 2013), influenza hemagglutinin (Isaacs et al., 2021), and human immunodeficiency virus glycoprotein 140 (Pancera et al., 2014) because the gp41 motif can be self-assembled into a stable six-helical bundle structure that is required to drive membrane fusion and cell entry of HIV-1 (Chan et al., 1997). This technique was also planned for use in MERS-CoV vaccine development funded by the CEPI. With the urgent

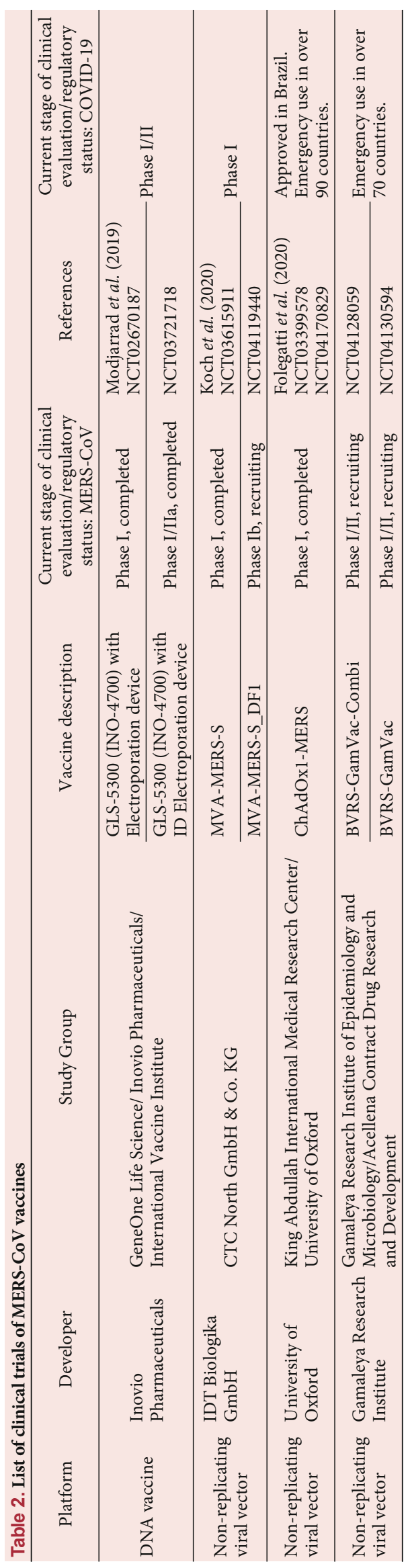




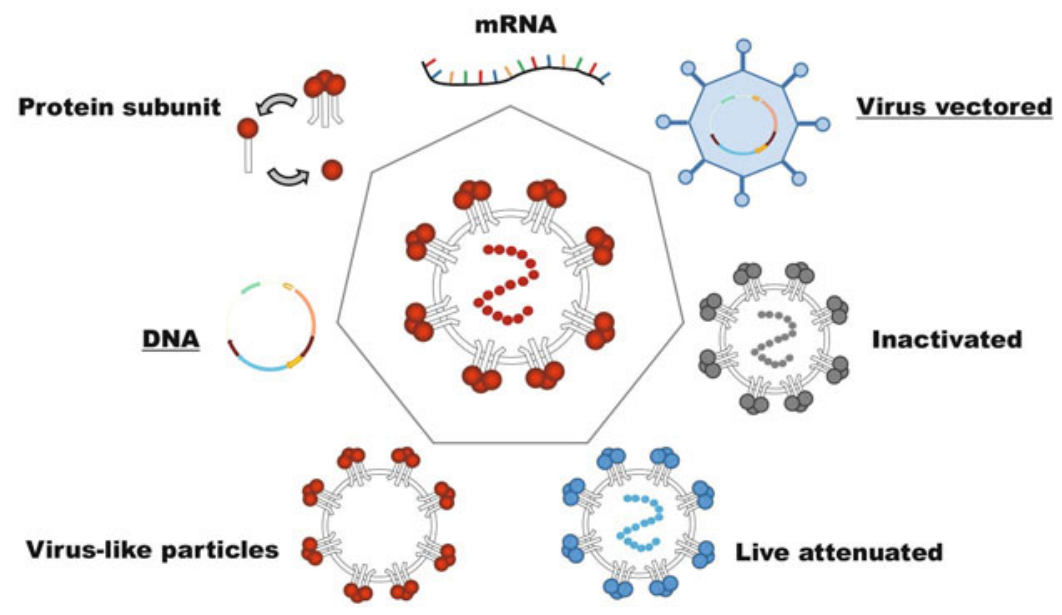

Fig. 2. Technologies used in vaccine development. DNA, mRNA, and virus-vectored vaccine coding antigen genes, protein subunit, virus-like particles, and inactivated and live attenuated viruses can be explored for novel vaccine development against emerging viral pathogens. Among these technologies, DNA and virus-vectored vaccines (with underline) with the full $\mathrm{S}$ gene are currently under clinical trials for MERS-CoV vaccine development.

need for the COVID-19 vaccine, however, this technique was quickly applied to the SARS-CoV-2 S to stabilize the authentic pre-fusion conformation that preserves neutralizing epitopes present on the virion surface. A clinical phase 1 study using this construct showed the limitation of the molecular clamp causing false positivity for $\mathrm{HIV}-1$ in normal individuals. Gp41 motif-induced antibodies cross-reacted with some HIV diagnostics, although there were robust and highly correlated humoral and cellular immune responses (Chappell et al., 2021). This false positivity led to abandon further clinical trials using gp41 as a trimerization motif. New clamp molecules need to be identified from other viruses or systems with similar levels of gp41 stability.

\section{Clinical Trial Status of MERS-CoV Vaccines}

All vaccine candidates described in the following clinical trial section used unmodified full-length $\mathrm{S}$ sequences (Table 2). In the absence of specific therapeutic agents, vaccination is expected to be an effective strategy for preventing MERS-CoV infection in both humans and animals. To date, several MERS$\mathrm{CoV}$ vaccine candidates have been developed using various platform technologies and are undergoing clinical trials, including DNA and recombinant viral vector-based vaccines such as adenoviral vectors, modified vaccinia virus Ankara, and recombinant measles virus (Graham et al., 2013; Modjarrad, 2016; Yong et al., 2019) (Fig. 2).

\section{MERS-CoV DNA vaccine}

DNA vaccines generally refer to vaccines that have DNA expressing an immunogenic protein placed in a plasmid vector and delivered into host cells. Usually, DNA vaccines are delivered to host cells by electroporation (Aihara and Miyazaki, 1998). Compared with other vaccine technologies, DNA vaccines have the advantage of rapid development, and they may be useful against new emerging infectious diseases (Sardesai and Weiner, 2011; Rauch et al., 2018). DNA vaccines also have the advantage of inducing both humoral and cell-mediated immune responses, but when used alone, immunogenicity is not strongly induced, and using an appropriate adjuvant is recommended (Liu, 2011). During the early stages of DNA vaccine development, there are concerns that DNA vaccines are incorporated into the host chromosome and they cause mutations in host cells, leading to tumor generation (Nichols et al., 1995; Rauch et al., 2018). However, after repeated clinical trials using DNA vaccines, it has been demonstrated that the probability of inducing host cell mutations is lower than that of spontaneous gene mutations (Sheets et al., 2006).

Vaccine development using DNA vaccine technology is also underway for MERS-CoV infection. In particular, the GLS5300 (INO-4700) MERS-CoV DNA vaccine, which contains the full-length $S$ gene, induces antigen-specific neutralizing antibodies in mice, monkeys, and camels, as well as strong cell-mediated immunity (Muthumani et al., 2015). In addition, it was confirmed that macaque monkeys vaccinated with GLS-5300 (INO-4700) MERS-CoV DNA vaccine showed strong protective immunity against wild-type MERS-CoV challenge, thereby improving lung pathology and reducing viral load in the lungs (Muthumani et al., 2015). Based on these pre-clinical experimental results, Phase 1 clinical trials of the GLS-5300 (INO-4700) MERS-CoV DNA vaccine have been completed (NCT02670187, NCT03721718). As a result, the GLS-5300 (INO-4700) DNA vaccine developed by Inovio Pharmaceuticals was confirmed to induce immunogenicity without severe side effects, and it was confirmed that serconversion by S1-ELISA was observed in $86 \%$ of clinical participants after the second immunization (Modjarrad et al., 2019). Inovio Pharmaceuticals developed INO-4800 (Smith et al., 2020), a candidate for a SARS-CoV-2 DNA vaccine with the same design as the MERS-CoV vaccine containing a fulllength $S$ gene, and it is currently in phase $1 / 2$ clinical trials (NCT04447781, NCT04336410).

\section{MERS-CoV viral vector vaccine}

Viral vector-based vaccines involve the use of a recombinant unrelated virus that contains the targeted antigen gene or protein as a vaccine. Viral vector-based vaccines are generally known to induce strong antigen-specific antibody responses and cell-mediated immune responses, which have the advantage of inducing sufficient immune responses without adjuvants (Rollier et al., 2011). In addition, viral vectors can easily insert large foreign genes, allowing flexibility in designing vaccine targets. However, the process of manufacturing the viral 
vector vaccine is more complicated than the process of manufacturing other vaccines, and it requires considerable technical skills to select and optimize cells that are efficient for virus production, so it can act as a disadvantage in terms of productivity (Rauch et al., 2018). In addition, most people already have antibodies against adenovirus or the measles virus; therefore, adenovirus or the measles virus can be incapacitated by the pre-existing antibodies when used as a viral vector. Therefore, the effectiveness of the vaccine may be reduced due to poor vaccine delivery efficiency. Therefore, it is necessary to choose a viral vector to avoid the interference by pre-existing antibodies for development of an effective vaccine (Knuchel et al., 2013; Wong et al., 2014).

Viral vector-based vaccines are also being studied for targeting S proteins, similar to DNA vaccines. Various viral vector vaccines are being used to develop MERS-CoV vaccines (Enjuanes et al., 2008; Schindewolf and Menachery, 2019), and among them, we take a closer look at the viral vectors currently undergoing clinical trials as MERS-CoV vaccines. The first vaccine uses the modified vaccinia virus Ankara (MVA) as a viral vector. The MVA-based MERS-CoV vaccine (MVA-MERS-S) contains a full-length $S$ protein as an antigen, similar to the DNA vaccine (Sutter and Moss, 1992; Stittelaar et al., 2001). The MVA-MERS-S vaccine has been found to induce antigen-specific neutralizing antibodies, as well as $\mathrm{CD}^{+} \mathrm{T}$ cell responses, in mice (Song et al., 2013). Furthermore, its protective immunity effect was confirmed using the wild-type MERS-CoV challenge test in hDPP4 transgenic mice (Volz et al., 2015); it was confirmed that inflammation and lymph node hyperplasia caused by viral infection were reduced (Langenmayer et al., 2018). Similarly, in dromedary camels, it was confirmed that antigen-specific neutralizing antibodies were induced after the MVA-MERS-S vaccination. In addition, it was confirmed that the release of infectious virions was effectively blocked when the nasal cavity was infected by wild-type MERS-CoV after the MVAMERS-S vaccination. Therefore, the MVA-MERS-S vaccine is considered to effectively control the transmission of MERS$\mathrm{CoV}$ from camels to humans (Haagmans et al., 2016). Based on the pre-clinical experimental results, Phase I clinical trials were conducted (NCT03615911), and the safety and immunogenicity of the MVA-MERS-S vaccine were confirmed in a phase 1 clinical trial (Koch et al., 2020). A phase 1 b clinical trial is underway to confirm the immune response by homologous prime-boost immunization in more volunteers (NCT04119440). Currently, Phase 1 clinical trials are in progress for the SARS-CoV-2 (MVA-SARS-2-ST) vaccine, which was developed using the same MVA as the vaccine vector used to develop the MERS-CoV vaccine (NCT04895449).

Adenovirus is a widely used platform for MERS-CoV vaccine studies. Adenovirus vectors have been used for a long time to develop vaccines against various diseases and have been used in HIV-1 vaccine clinical trials (Hammer et al., 2013). The effectiveness of the immunogenicity of the antigen translated to a protein from the adenovirus vector itself is not bad, but it is known that the immune response of the adenovirus vector vaccine is reduced by the pre-existing antibodies against the adenovirus in most people (Chirmule et al., 1999; Mercier et al., 2004; Mast et al., 2010). The adenovirus type 5-based vaccine, which is related to the development of the MERS-
$\mathrm{CoV}$ vaccine, induced antigen-specific neutralizing antibodies in mice, but protective immune responses were not confirmed in hDPP4 transgenic mice. In addition, humoral immune responses, as well as cell-mediated immune responses, have been reported in camels administered the same vaccine (Guo et al., 2015; Jung et al., 2018).

To avoid pre-existing antibodies, vaccine development using low prevalence vectors in humans, such as adenovirus type 26 or other animal origin adenoviruses such as chimpanzee adenovirus, and the heterologous prime-boost method using various types of adenoviruses is being explored. The BVRS-GamVac-Combi vaccine, a heterologous prime-boost immune vaccine using recombinant adenovirus type 26 and recombinant adenovirus type 5, induced high titers of antigen-specific neutralizing antibodies in mice (Dolzhikova et al., 2020). It was subsequently confirmed that the antibody titer was maintained for up to 18 months. In an experiment to confirm the protective efficacy of hDPP4 transgenic mice, the protective immune response against the lethal MERS$\mathrm{CoV}$ infection was confirmed, and the protective immunity was maintained even 7 months after the last immunization (Dolzhikova et al., 2020). Currently, phase $1 / 2$ clinical trials using the BVRS-GamVac-Combi vaccine are in progress, and the results of clinical trials are expected to be announced in December 2021 (NCT04128059, NCT04130594). The vaccine against SARS-CoV-2, which uses the same platform as the BVRS-GamVac-Combi vaccine, has also been developed and is being used as an emergency vaccine under the name of Sputnik V (Gam-Covid-Vac) in more than 70 countries, including Russia.

Chimpanzee adenoviruses are being explored to overcome pre-existing antibodies against adenovirus in humans. In the development of the MERS-CoV vaccine, the ChAdOx1MERS vaccine based on the chimpanzee adenovirus vector (ChAdOx1) was found to induce high titers of antigen-specific neutralizing antibodies, as well as a high cell-mediated immune response, in mice. In a hDPP4 transgenic mouse study, the ChAdOx1-MERS vaccine showed high protective efficacy against wild-type MERS-CoV lethal infection (Alharbi et al., 2017; Munster et al., 2017). It was confirmed that the ChAdOxl-MERS vaccine significantly reduced the viral load caused by the wild-type MERS-CoV virus infection in dromedary camels and also reduced the viral load in monkeys and showed a protective immune response against wild-type MERS-CoV virus infections (Alharbi et al., 2019; van Doremalen et al., 2020). Based on pre-clinical experimental results, Phase 1 clinical trials were conducted using the ChAdOx1MERS vaccine (NCT03399578, NCT04170829), and the results confirmed that ChAdOxl-MERS was safe and an immune response was well-induced for all test doses (Folegatti et al., 2020). The ChAdOx1 virus vector-based SARS-CoV-2 vaccine has been used as an emergency vaccine in more than 90 countries (Folegatti et al., 2020). It was developed by the University of Oxford research team, which is also the developer of ChAdOx1-MERS, and officially approved in Brazil under the name AZD1222 (or ChAdOx1-nCoV-19).

Several MERS-CoV vaccines based on viral vectors, such as adenovirus (Jung et al., 2018), Venezuelan equine encephalitis virus (Walls et al., 2017; Agnihothram et al., 2018), vesicular stomatitis virus (VSV) (Liu et al., 2018), measles 
virus (Malczyk et al., 2015), Newcastle disease virus (Liu et al., 2017), and rabies virus (Wirblich et al., 2017), have been developed. Additionally, various viral vectors, in addition to the viral vectors described above, are being explored in ongoing MERS-CoV vaccine development.

Four vaccines are currently undergoing clinical trials for MERS-CoV, which include a DNA vaccine (INO-4700) and the three virus vector-based vaccines as described above. The vaccine platforms used in clinical trials for the MERS-CoV vaccine have also been used to develop SARS-CoV-2 vaccines. Among them, two vaccines (a heterologous prime-boost immune vaccine using recombinant adenovirus type 26 and recombinant adenovirus type 5 and chimpanzee adenovirus vector [ChAdOxl] vaccine) have been developed and are being used as emergency vaccines in several countries ( $\mathrm{Li}$ et al., 2020).

\section{MERS-CoV inactivated, live attenuated and protein-based vaccine development}

Traditional inactivated vaccines (Agrawal et al., 2016; Deng et al., 2018), live attenuated vaccines (Almazán et al., 2013), and recombinant protein-based vaccines (Coleman et al., 2017; Jiaming et al., 2017) have also been explored for MERS$\mathrm{CoV}$, but most are still in the pre-clinical experimental stage.

Inactivated vaccines are made from virions, which are usually killed by chemical or radiational inactivation ( $\mathrm{Li}$ et al., 2020). In general, inactivated vaccine is safer than the attenuated vaccine, but has the disadvantage that it may be accompanied by structural change during the inactivation process (Bolles et al., 2011; Tseng et al., 2012). Various studies have been conducted to develop an inactivated MERS-CoV vaccine (Agrawal et al., 2016; Deng et al., 2018). Among such previous studies, gamma-irradiated MERS-CoV vaccine administered with alum or MF59 adjuvant induced neutralizing antibodies in mice. However, it has been confirmed that wild-type MERS-CoV infection after vaccination causes eosinophil-related lung pathology in mice (Agrawal et al., 2016). In contrast, a recent study found that the alum adjuvanted formalin-inactivated MERS-CoV vaccine prevented eosinophil-related lung pathology (Deng et al., 2018). Currently, there are no ongoing clinical trials for an inactivated MERSCoV.

The live attenuated vaccine, which utilizes the actual virus from which the pathogenicity has been removed, is the most immunogenic vaccine, and has been applied to prevent various infectious diseases (Minor, 2015). However, it was pointed out as a disadvantage that there are always opportunities for the pathogenicity to be recovered, which can cause infection in immunocompromised patients (Minor, 2015). In the development of the MERS-CoV vaccine, the live attenuated vaccine efficacy was confirmed in mice (Almazán et al., 2013), but it did not progress to clinical trials.

Recombinant protein-based vaccine is composed of a recombinant protein containing one or more antigenic proteins, and has the advantage of easy production and high safety (Hansson et al., 2000). Regarding the development of the MERS-CoV vaccine, a vaccine using the RBD or full $S$ protein was developed, and it was confirmed that the neutralizing antibody titer was highly induced in mice, and the protective immunity effect was also confirmed in hDPP4 mice
(Coleman et al., 2014, 2017; Tai et al., 2016; Jiaming et al., 2017). However, no recombinant protein-based vaccine has entered into clinical trials yet.

\section{Conclusion}

Vaccine development should be prioritized for MERS-CoV considering its CFR and the absence of a therapeutic agent. Several MERS-CoV vaccines using different platform technologies, such as DNA and viral vectors, are enrolled in clinical studies. However, most MERS-CoV vaccine clinical trials remain in the early phase $1 / 2$ studies although they were initiated earlier than trials for SARS-CoV-2. Furthermore, the platform technology targeted at MERS-CoV vaccine development is being used successfully for SARS-CoV-2 vaccines.

Given the nature of RNA viruses, a variant $S$ can emerge during the transmission of MERS-CoV from animals to humans or humans to humans. In contrast to monoclonal antibodies that cannot neutralize pseudoviruses even with one amino acid mutation in S (Goo et al., 2020), a vaccine targeting one viral strain $S$ can induce cross-protective immune responses against multiple viral strains with mutation in S (Choi et al., 2020). However, if a new $S$ variant escapes the neutralizing antibodies developed in the original MERS-CoV vaccines, it should be possible to quickly produce a vaccine against a mutant strain using the identified viral sequence once the platform technology is established. To induce more efficacious neutralizing antibodies, the pre-fusion form of S-like COVID19 vaccines developed by Moderna, Pfizer/BioNTech, and Jassen need to be adopted for MERS-CoV vaccines (Dai and Gao, 2021).

Given the cases of other vaccine licenses such as the Hepatitis B vaccine (Eyigun et al., 1998), the evaluation of vaccine efficacy can be made easier if the immune correlate of protection is identified. Using the WHO International Standard for MERS-CoV antibodies released in 2020, the pooling of sera from MERS-recovered patients can be a useful tool for harmonizing the assessment of immunogenicity, especially binding and neutralizing antibody responses to different vaccine platforms. This approach was established by the International Vaccine Institute, NIBSC, and funded by CEPI (WHO/BS/2020.2398 Establishment of 1st WHO International Standard for anti-MERS-CoV antibody). In addition, further studies are needed to determine the critical cellular immune responses induced by vaccines against MERS-CoV infection. Considering the characteristic immune responses induced by each platform technique, heterologous priming/ boosting may also be considered to maximize protective immunity (Choi et al., 2020).

In conclusion, the platform technologies tested during MERS$\mathrm{CoV}$ vaccine development can be used for other unknown emerging pathogens in the future.

\section{Acknowledgements}

This work was supported by grants of the Korea Health Technology R\&D Project through the Korea Health Industry Development Institute (KHIDI), funded by the Ministry of 
Health \& Welfare, Republic of Korea (grant number : HV20C0150 and HD17A0085).

\section{Conflicts of Interest}

The authors declare they have no potential conflicts of interest to disclose.

\section{References}

Agnihothram, S., Menachery, V.D., Yount, B.L.Jr., Lindesmith, L.C., Scobey, T., Whitmore, A., Schäfer, A., Heise, M.T., and Baric, R.S. 2018. Development of a broadly accessible venezuelan equine encephalitis virus replicon particle vaccine platform. J. Virol. 92, e00027-18.

Agrawal, A.S., Tao, X., Algaissi, A., Garron, T., Narayanan, K., Peng, B.H., Couch, R.B., and Tseng, C.T. 2016. Immunization with inactivated middle east respiratory syndrome coronavirus vaccine leads to lung immunopathology on challenge with live virus. Hum. Vaccin Immunother. 12, 2351-2356.

Aihara, H. and Miyazaki, J. 1998. Gene transfer into muscle by electroporation in vivo. Nat. Biotechnol. 16, 867-870.

Al-Amri, S.S., Abbas, A.T., Siddiq, L.A., Alghamdi, A., Sanki, M.A., Al-Muhanna, M.K., Alhabbab, R.Y., Azhar, E.I., Li, X., and Hashem, A.M. 2017. Immunogenicity of candidate MERS-CoV DNA vaccines based on the spike protein. Sci. Rep. 7, 44875.

Alharbi, N.K., Padron-Regalado, E., Thompson, C.P., Kupke, A., Wells, D., Sloan, M.A., Grehan, K., Temperton, N., Lambe, T., Warimwe, G., et al. 2017. ChAdOx1 and MVA based vaccine candidates against MERS-CoV elicit neutralising antibodies and cellular immune responses in mice. Vaccine 35, 3780-3788.

Alharbi, N.K., Qasim, I., Almasoud, A., Aljami, H.A., Alenazi, M.W., Alhafufi, A., Aldibasi, O.S., Hashem, A.M., Kasem, S., Albrahim, R., et al. 2019. Humoral immunogenicity and efficacy of a single dose of chadox 1 mers vaccine candidate in dromedary camels. Sci. Rep. 9, 16292.

Almazán, F., DeDiego, M.L., Sola, I., Zuñiga, S., Nieto-Torres, J.L., Marquez-Jurado, S., Andrés, G., and Enjuanes, L. 2013. Engineering a replication-competent, propagation-defective Middle East Respiratory Syndrome coronavirus as a vaccine candidate. $m B i o$ 4, e00650-13.

Bernasconi, V., Kristiansen, P.A., Whelan, M., Román, R.G., Bettis, A., Yimer, S.A., Gurry, C., Andersen, S.R., Yeskey, D., Mandi, H., et al. 2020. Developing vaccines against epidemic-prone emerging infectious diseases. Bundesgesundheitsbl. 63, 65-73.

Bhat, E.A., Khan, J., Sajjad, N., Ali, A., Aldakeel, F.M., Mateen, A., Alqahtani, M.S., and Syed, R. 2021. SARS-CoV-2: Insight in genome structure, pathogenesis and viral receptor binding analysis - an updated review. Int. Immunopharmacol. 95, 107493.

Bolles, M., Deming, D., Long, K., Agnihothram, S., Whitmore, A., Ferris, M., Funkhouser, W., Gralinski, L., Totura, A., Heise, M., et al.2011. A double-inactivated severe acute respiratory syndrome coronavirus vaccine provides incomplete protection in mice and induces increased eosinophilic proinflammatory pulmonary response upon challenge. J. Virol. 85, 12201-12215.

Bosch, B.J., van der Zee, R., de Haan, C.A., and Rottier, P.J.M. 2003. The coronavirus spike protein is a class I virus fusion protein: structural and functional characterization of the fusion core complex. J. Virol. 77, 8801-8811.

Chan, D.C., Fass, D., Berger, J.M., and Kim, P.S. 1997. Core structure of gp41 from the HIV envelope glycoprotein. Cell 89, 263-273.

Chappell, K.J., Mordant, F.L., Li, Z., Wijesundara, D.K., Ellenberg, P., Lackenby, J.A., Cheung, S.T.M., Modhiran, N., Avumegah, M.S.,
Henderson, C.L., et al. 2021. Safety and immunogenicity of an MF59-adjuvanted spike glycoprotein-clamp vaccine for SARSCoV-2: a randomised, double-blind, placebo-controlled, phase 1 trial. Lancet Infect. Dis. 21, 1383-1394.

Chen, Y., Liu, Q., and Guo, D. 2020. Emerging coronaviruses: genome structure, replication, and pathogenesis. J. Med. Virol. 92, 418423.

Chirmule, N., Propert, K., Magosin, S., Qian, Y., Qian, R., and Wilson, J. 1999. Immune responses to adenovirus and adeno-associated virus in humans. Gene Ther. 6, 1574-1583.

Cho, S.Y., Kang, J.M., Ha, Y.E., Park, G.E., Lee, J.Y., Ko, J.H., Kim, J.M., Kang, C.I., Jo, I.J., Ryu, J.G., et al. 2016. MERS-CoV outbreak following a single patient exposure in an emergency room in South Korea: an epidemiological outbreak study. Lancet 388, $994-$ 1001.

Choi, J.A., Goo, J., Yang, E., Jung, D.I., Lee, S., Rho, S., Jeong, Y., Park, Y.S., Park, H., Moon, Y.H., et al. 2020. Cross-protection against MERS-CoV by prime-boost vaccination using viral spike DNA and protein. J. Virol. 94, e01176-20.

Coleman, C.M., Liu, Y.V., Mu, H., Taylor, J.K., Massare, M., Flyer, D.C., Smith, G.E., and Frieman, M.B. 2014. Purified coronavirus spike protein nanoparticles induce coronavirus neutralizing antibodies in mice. Vaccine 32, 3169-3174.

Coleman, C.M., Venkataraman, T., Liu, Y.V., Glenn, G.M., Smith, G.E., Flyer, D.C., and Frieman, M.B. 2017. MERS-CoV spike nanoparticles protect mice from MERS-CoV infection. Vaccine 35, 1586-1589.

Dai, L. and Gao, G.F. 2021. Viral targets for vaccines against COVID19. Nat. Rev. Immunol. 21, 73-82.

de Groot, R.J., Baker, S.C., Baric, R.S., Brown, C.S., Drosten, C., Enjuanes, L., Fouchier, R.A.M., Galiano, M., Gorbalenya, A.E., Memish, Z.A., et al. 2013. Middle East respiratory syndrome coronavirus (MERS-CoV): Announcement of the coronavirus study group. J. Virol. 87, 7790-7792.

Deng, Y., Lan, J., Bao, L., Huang, B., Ye, F., Chen, Y., Yao, Y., Wang, W., Qin, C., and Tan, W. 2018. Enhanced protection in mice induced by immunization with inactivated whole viruses compare to spike protein of middle east respiratory syndrome coronavirus. Emerg. Microbes Infect. 7, 60.

Dolzhikova, I.V., Grousova, D.M., Zubkova, O.V., Tukhvatulin, A.I., Kovyrshina, A.V., Lubenets, N.L., Ozharovskaia, T.A., Popova, O., Esmagambetov, I.B., Shcheblyakov, D.V., et al. 2020. Preclinical studies of immunogenity, protectivity, and safety of the combined vector vaccine for prevention of the Middle East respiratory syndrome. Acta Naturae 12, 114-123.

Drosten, C., Meyer, B., Müller, M.A., Corman, V.M., Al-Masri, M., Hossain, R., Madani, H., Sieberg, A., Bosch, B.J., Lattwein, E., et al. 2014. Transmission of MERS-coronavirus in household contacts. N. Engl. J. Med. 371, 828-835.

Du, L., Zhao, G., Kou, Z., Ma, C., Sun, S., Poon, V.K.M., Lu, L., Wang, L., Debnath, A.K., Zheng, B.J., et al. 2013. Identification of a receptor-binding domain in the s protein of the novel human coronavirus middle east respiratory syndrome coronavirus as an essential target for vaccine development. J. Virol. 87, 9939-9942.

Enjuanes, L., Dediego, M.L., Alvarez, E., Deming, D., Sheahan, T., and Baric, R. 2008. Vaccines to prevent severe acute respiratory syndrome coronavirus-induced disease. Virus Res. 133, 45-62.

Eyigun, C.P., Yilmaz, S., Gül, C., Sengül, A., Hacibektasoglu, A., and Van Thiel, D.H. 1998. A comparative trial of two surface subunit recombinant hepatitis B vaccines vs a surface and pres subunit vaccine for immunization of healthy adults. J. Viral Hepat. 5, 265-269.

Folegatti, P.M., Bittaye, M., Flaxman, A., Lopez, F.R., Bellamy, D., Kupke, A., Mair, C., Makinson, R., Sheridan, J., Rohde, C., et al. 2020. Safety and immunogenicity of a candidate Middle East respiratory syndrome coronavirus viral-vectored vaccine: a doseescalation, open-label, non-randomised, uncontrolled, phase 1 
trial. Lancet Infect. Dis. 20, 816-826.

Goo, J., Jeong, Y., Park, Y.S., Yang, E., Jung, D.I., Rho, S., Park, U., Sung, H., Park, P.G., Choi, J.A., et al. 2020. Characterization of novel monoclonal antibodies against MERS-coronavirus spike protein. Virus Res. 278, 197863.

Gouglas, D., Christodoulou, M., Plotkin, S.A., and Hatchett, R. 2019. CEPI: Driving progress toward epidemic preparedness and response. Epidemiol. Rev. 41, 28-33.

Graham, R.L., Donaldson, E.F., and Baric, R.S. 2013. A decade after SARS: strategies for controlling emerging coronaviruses. Nat. Rev. Microbiol. 11, 836-848.

Guo, X., Deng, Y., Chen, H., Lan, J., Wang, W., Zou, X., Hung, T., Lu, Z., and Tan, W. 2015. Systemic and mucosal immunity in mice elicited by a single immunization with human adenovirus type 5 or 41 vector-based vaccines carrying the spike protein of Middle East respiratory syndrome coronavirus. Immunology 145, 476-484.

Haagmans, B.L., van den Brand, J.M.A., Raj, V.S., Volz, A., Wohlsein, P., Smits, S.L., Schipper, D., Bestebroer, T.M., Okba, N., Fux, R., et al. 2016. An orthopoxvirus-based vaccine reduces virus excretion after MERS-CoV infection in dromedary camels. Science 351, 77-81.

Hammer, S.M., Sobieszczyk, M.E., Janes, H., Karuna, S.T., Mulligan, M.J., Grove, D., Koblin, B.A., Buchbinder, S.P., Keefer, M.C., Tomaras, G.D., et al. 2013. Efficacy trial of a DNA/rAd5 HIV-1 preventive vaccine. N. Engl. J. Med. 369, 2083-2092.

Hansson, M., Nygren, P.A., and Ståhl, S. 2000. Design and production of recombinant subunit vaccines. Biotechnol. Appl. Biochem. 32, 95-107.

Hemida, M.G., Elmoslemany, A., Al-Hizab, F., Alnaeem, A., Almathen, F., Faye, B., Chu, D.K.W., Perera, R.A.P.M., and Peiris, M. 2017. Dromedary camels and the transmission of Middle East respiratory syndrome coronavirus (MERS-CoV). Transbound. Emerg. Dis. 64, 344-353.

Hulswit, R.J.G., Lang, Y., Bakkers, M.J.G., Li, W., Li, Z., Schouten, A., Ophorst, B., van Kuppeveld, F.J.M., Boons, G.J., Bosch, B.J., et al. 2019. Human coronaviruses OC43 and HKU1 bind to 9$O$-acetylated sialic acids via a conserved receptor-binding site in spike protein domain A. Proc. Natl. Acad. Sci. USA 116, 26812690.

Isaacs, A., Li, Z., Cheung, S.T.M., Wijesundara, D.K., McMillan, C.L.D., Modhiran, N., Young, P.R., Ranasinghe, C., Watterson, D., and Chappell, K.J. 2021. Adjuvant selection for influenza and RSV prefusion subunit vaccines. Vaccines $\mathbf{9}, 71$.

Jiaming, L., Yanfeng, Y., Yao, D., Yawei, H., Linlin, B., Baoying, H., Jinghua, Y., Gao, G.F., Chuan, Q., and Wenjie, T. 2017. The recombinant $\mathrm{n}$-terminal domain of spike proteins is a potential vaccine against Middle East respiratory syndrome coronavirus (MERS-CoV) infection. Vaccine 35, 10-18.

Jung, S.Y., Kang, K.W., Lee, E.Y., Seo, D.W., Kim, H.L., Kim, H., Kwon, T., Park, H.L., Kim, H., Lee, S. M., et al. 2018. Heterologous prime-boost vaccination with adenoviral vector and protein nanoparticles induces both Th1 and Th2 responses against middle east respiratory syndrome coronavirus. Vaccine 36, 3468-3476.

Kashte, S., Gulbake, A., El-Amin Iii, S.F., and Gupta, A. 2021. COVID19 vaccines: rapid development, implications, challenges and future prospects. Hum. Cell 34, 711-733.

Knuchel, M.C., Marty, R.R., Morin, T.N., Ilter, O., Zuniga, A., and Naim, H.Y. 2013. Relevance of a pre-existing measles immunity prior immunization with a recombinant measles virus vector. Hum. Vaccin. Immunother. 9, 599-606.

Koch, T., Dahlke, C., Fathi, A., Kupke, A., Krähling, V., Okba, N.M.A., Halwe, S., Rohde, C., Eickmann, M., Volz, A., et al. 2020. Safety and immunogenicity of a modified vaccinia virus Ankara vector vaccine candidate for Middle East respiratory syndrome: an openlabel, phase 1 trial. Lancet Infect. Dis. 20, 827-838.

Langenmayer, M.C., Lülf-Averhoff, A.T., Adam-Neumair, S., Fux,
R., Sutter, G., and Volz, A. 2018. Distribution and absence of generalized lesions in mice following single dose intramuscular inoculation of the vaccine candidate MVA-MERS-S. Biologicals 54, $58-62$.

Li, Y.D., Chi, W.Y., Su, J.H., Ferrall, L., Hung, C.F., and Wu, T.C. 2020. Coronavirus vaccine development: from SARS and MERS to COVID-19. J. Biomed. Sci. 27, 104.

Liu, M.A. 2011. DNA vaccines: an historical perspective and view to the future. Immunol. Rev. 239, 62-84.

Liu, R.Q., Ge, J.Y., Wang, J.L., Shao, Y., Zhang, H.L., Wang, J.L., Wen, Z.Y., and Bu, Z.G. 2017. Newcastle disease virus-based MERS$\mathrm{CoV}$ candidate vaccine elicits high-level and lasting neutralizing antibodies in bactrian camels. J Integr Agric. 16, 2264-2273.

Liu, R., Wang, J., Shao, Y., Wang, X., Zhang, H., Shuai, L., Ge, J., Wen, Z., and Bu, Z. 2018. A recombinant VSV-vectored MERS-CoV vaccine induces neutralizing antibody and $\mathrm{T}$ cell responses in rhesus monkeys after single dose immunization. Antiviral Res. 150, 30-38.

Malczyk, A.H., Kupke, A., Prüfer, S., Scheuplein, V.A., Hutzler, S., Kreuz, D., Beissert, T., Bauer, S., Hubich-Rau, S., Tondera, C., et al. 2015. A highly immunogenic and protective Middle East respiratory syndrome coronavirus vaccine based on a recombinant measles virus vaccine platform. J. Virol. 89, 11654-11667.

Maslow, J.N. 2018. The cost and challenge of vaccine development for emerging and emergent infectious diseases. Lancet Glob. Health 6, e1266-e1267.

Mast, T.C., Kierstead, L., Gupta, S.B., Nikas, A.A., Kallas, E.G., Novitsky, V., Mbewe, B., Pitisuttithum, P., Schechter, M., Vardas, E., et al. 2010. International epidemiology of human pre-existing adenovirus (Ad) type-5, type-6, type-26 and type-36 neutralizing antibodies: correlates of high Ad5 titers and implications for potential HIV vaccine trials. Vaccine 28, 950-957.

McLellan, J.S., Chen, M., Joyce, M.G., Sastry, M., Stewart-Jones, G.B.E., Yang, Y., Zhang, B., Chen, L., Srivatsan, S., Zheng, A., et al. 2013. Structure-based design of a fusion glycoprotein vaccine for respiratory syncytial virus. Science 342, 592-598.

Mehand, M.S., Al-Shorbaji, F., Millett, P., and Murgue, B. 2018. The WHO R\&D Blueprint: 2018 review of emerging infectious diseases requiring urgent research and development efforts. Antiviral Res. 159, 63-67.

Memish, Z.A., Perlman, S., Van Kerkhove, M.D., and Zumla, A. 2020. Middle East respiratory syndrome. Lancet 395, 1063-1077.

Mercier, G.T., Campbell, J.A., Chappell, J.D., Stehle, T., Dermody, T.S., and Barry, M.A. 2004. A chimeric adenovirus vector encoding reovirus attachment protein sigmal targets cells expressing junctional adhesion molecule 1. Proc. Natl. Acad. Sci. USA 101, 6188-6193.

Minor, P.D. 2015. Live attenuated vaccines: historical successes and current challenges. Virology 479-480, 379-392.

Modjarrad, K. 2016. MERS-CoV vaccine candidates in development: the current landscape. Vaccine 34, 2982-2987.

Modjarrad, K., Roberts, C.C., Mills, K.T., Castellano, A.R., Paolino, K., Muthumani, K., Reuschel, E.L., Robb, M.L., Racine, T., Oh, M.D., et al. 2019. Safety and immunogenicity of an anti-Middle East respiratory syndrome coronavirus DNA vaccine: a phase 1, open-label, single-arm, dose-escalation trial. Lancet Infect. Dis. 19, 1013-1022.

Mohd, H.A., Al-Tawfiq, J.A., and Memish, Z.A. 2016. Middle east respiratory syndrome coronavirus (MERS-CoV) origin and animal reservoir. Virol. J. 13, 87.

Munster, V.J., Wells, D., Lambe, T., Wright, D., Fischer, R.J., Bushmaker, T., Saturday, G., van Doremalen, N., Gilbert, S.C., de Wit, E., et al. 2017. Protective efficacy of a novel simian adenovirus vaccine against lethal MERS-CoV challenge in a transgenic human DPP4 mouse model. npj Vaccines 2, 28.

Muthumani, K., Falzarano, D., Reuschel, E.L., Tingey, C., Flingai, S., Villarreal, D.O., Wise, M., Patel, A., Izmirly, A., Aljuaid, A., 
et al. 2015. A synthetic consensus anti-spike protein DNA vaccine induces protective immunity against Middle East respiratory syndrome coronavirus in nonhuman primates. Sci. Transl. Med. 7, 301ra132.

Nichols, W.W., Ledwith, B.J., Manam, S.V., and Troilo, P.J. 1995. Potential DNA vaccine integration into host cell genome. Ann. N. Y. Acad. Sci. 772, 30-39.

Pallesen, J., Wang, N., Corbett, K.S., Wrapp, D., Kirchdoerfer, R.N., Turner, H.L., Cottrell, C.A., Becker, M.M., Wang, L., Shi, W., et al. 2017. Immunogenicity and structures of a rationally designed prefusion MERS-CoV spike antigen. Proc. Natl. Acad. Sci. USA 114, E7348-E7357.

Pancera, M., Zhou, T., Druz, A., Georgiev, I.S., Soto, C., Gorman, J., Huang, J., Acharya, P., Chuang, G.Y., Ofek, G., et al. 2014. Structure and immune recognition of trimeric pre-fusion HIV-1 Env. Nature 514, 455-461.

Plotkin, S.A. 2017. Vaccines for epidemic infections and the role of CEPI. Hum. Vaccin. Immunother. 13, 2755-2762.

Rauch, S., Jasny, E., Schmidt, K.E., and Petsch, B. 2018. New vaccine technologies to combat outbreak situations. Front. Immunol. 9, 1963.

Rollier, C.S., Reyes-Sandoval, A., Cottingham, M.G., Ewer, K., and Hill, A.V. 2011. Viral vectors as vaccine platforms: deployment in sight. Curr. Opin. Immunol. 23, 377-382.

Røttingen, J.A., Gouglas, D., Feinberg, M., Plotkin, S., Raghavan, K.V., Witty, A., Draghia-Akli, R., Stoffels, P., and Piot, P. 2017. New vaccines against epidemic infectious diseases. N. Engl. J. Med. 376, 610-613.

Sardesai, N.Y. and Weiner, D.B. 2011. Electroporation delivery of DNA vaccines: prospects for success. Curr. Opin. Immunol. 23, 421-429.

Schindewolf, C. and Menachery, V.D. 2019. Middle East respiratory syndrome vaccine candidates: cautious optimism. Viruses 11, 74.

Sheets, R.L., Stein, J., Manetz, T.S., Duffy, C., Nason, M., Andrews, C., Kong, W.P., Nabel, G.J., and Gomez, P.L. 2006. Biodistribution of DNA plasmid vaccines against HIV-1, Ebola, severe acute respiratory syndrome, or west nile virus is similar, without integration, despite differing plasmid backbones or gene inserts. Toxicol. Sci. 91, 610-619.

Smith, T.R.F., Patel, A., Ramos, S., Elwood, D., Zhu, X., Yan, J., Gary, E.N., Walker, S.N., Schultheis, K., Purwar, M., et al. 2020. Immunogenicity of a DNA vaccine candidate for COVID-19. Nat. Commun. 11, 2601.

Song, F., Fux, R., Provacia, L.B., Volz, A., Eickmann, M., Becker, S., Osterhaus, A.D., Haagmans, B.L., and Sutter, G. 2013. Middle East respiratory syndrome coronavirus spike protein delivered by modified vaccinia virus Ankara efficiently induces virus-neutralizing antibodies. J. Virol. 87, 11950-11954.

Stittelaar, K.J., Kuiken, T., de Swart, R.L., van Amerongen, G., Vos, H.W., Niesters, H.G., van Schalkwijk, P., van der Kwast, T., Wyatt, L.S., Moss, B., et al. 2001. Safety of modified vaccinia virus Ankara (MVA) in immune-suppressed macaques. Vaccine 19, 3700-3709.

Sutter, G. and Moss, B. 1992. Nonreplicating vaccinia vector efficiently expresses recombinant genes. Proc. Natl. Acad. Sci. USA 89, 10847-10851.

Tai, W., Zhao, G., Sun, S., Guo, Y., Wang, Y., Tao, X., Tseng, C.T.K., Li, F., Jiang, S., Du, L., et al. 2016. A recombinant receptor-binding domain of MERS-CoV in trimeric form protects human di- peptidyl peptidase 4 (hDPP4) transgenic mice from MERS-CoV infection. Virology 499, 375-382.

Tseng, C.T., Sbrana, E., Iwata-Yoshikawa, N., Newman, P.C., Garron, T., Atmar, R.L., Peters, C.J., and Couch, R.B. 2012. Immunization with SARS coronavirus vaccines leads to pulmonary immunopathology on challenge with the SARS virus. PLoS ONE 7, e35421. van Boheemen, S., de Graaf, M., Lauber, C., Bestebroer, T.M., Raj, V.S., Zaki, A.M., Osterhaus, A.D.M.E., Haagmans, B.L., Gorbalenya, A.E., Snijder, E.J., et al. 2012. Genomic characterization of a newly discovered coronavirus associated with acute respiratory distress syndrome in humans. mBio 3, e00473-12.

van Doremalen, N., Haddock, E., Feldmann, F., Meade-White, K., Bushmaker, T., Fischer, R.J., Okumura, A., Hanley, P.W., Saturday, G., Edwards, N.J., et al. 2020. A single dose of ChAdOx1 MERS provides protective immunity in rhesus macaques. Sci. Adv. 6, eaba8399.

Vandeputte, J., Saville, M., Cavaleri, M., Friede, M., Hacker, A., Mueller, S.O., Rizzi, R., Smith, D., Thirstrup, S., Wagner, R., et al. 2021. IABS/CEPI platform technology webinar: is it possible to reduce the vaccine development time? Biologicals. 71, 55-60.

Volz, A., Kupke, A., Song, F., Jany, S., Fux, R., Shams-Eldin, H., Schmidt, J., Becker, C., Eickmann, M., Becker, S., et al. 2015. Protective efficacy of recombinant modified vaccinia virus Ankara delivering Middle East respiratory syndrome coronavirus spike glycoprotein. J. Virol. 89, 8651-8656.

Walls, A.C., Tortorici, M.A., Snijder, J., Xiong, X., Bosch, B.J., Rey, F.A., and Veesler, D. 2017. Tectonic conformational changes of a coronavirus spike glycoprotein promote membrane fusion. Proc. Natl. Acad. Sci. USA 114, 11157-11162.

Wang, Q., Qi, J., Yuan, Y., Xuan, Y., Han, P., Wan, Y., Ji, W., Li, Y., Wu, Y., Wang, J., et al. 2014. Bat origins of MERS-CoV supported by bat coronavirus HKU4 usage of human receptor CD26. Cell Host Microbe 16, 328-337.

Wang, N., Shi, X., Jiang, L., Zhang, S., Wang, D., Tong, P., Guo, D., Fu, L., Cui, Y., Liu, X., et al. 2013. Structure of MERS-CoV spike receptor-binding domain complexed with human receptor DPP4. Cell Res. 23, 986-993.

Wirblich, C., Coleman, C.M., Kurup, D., Abraham, T.S., Bernbaum, J.G., Jahrling, P.B., Hensley, L.E., Johnson, R.F., Frieman, M.B., and Schnell, M.J. 2017. One-health: a safe, efficient, dual-use vaccine for humans and animals against Middle East respiratory syndrome coronavirus and rabies virus. J. Virol. 91, e02040-16.

Wong, G., Audet, J., Fernando, L., Fausther-Bovendo, H., Alimonti, J.B., Kobinger, G.P., and Qiu, X. 2014. Immunization with vesicular stomatitis virus vaccine expressing the Ebola glycoprotein provides sustained long-term protection in rodents. Vaccine 32, $5722-5729$.

Wong, G. and Qiu, X. 2018. Funding vaccines for emerging infectious diseases. Hum. Vaccin. Immunother. 14, 1760-1762.

Yong, C.Y., Ong, H.K., Yeap, S.K., Ho, K.L., and Tan, W.S. 2019. Recent advances in the vaccine development against Middle East respiratory syndrome-coronavirus. Front. Microbiol. 10, 1781.

Yu, X., Zhang, S., Jiang, L., Cui, Y., Li, D., Wang, D., Wang, N., Fu, L., Shi, X., Li, Z., et al. 2015. Structural basis for the neutralization of MERS-CoV by a human monoclonal antibody MERS-27. Sci. Rep. 5, 13133.

Zumla, A., Hui, D.S., and Perlman, S. 2015. Middle east respiratory syndrome. Lancet 386, 995-1007. 